\title{
An improved urban cellular automata model by using the trend-adjusted neighborhood
}

\author{
Xuecao Li, Yuyu Zhou* (iD and Wei Chen
}

\begin{abstract}
Background: Cellular automata (CA)-based models have been extensively used in urban sprawl modeling. Presently, most studies focused on the improvement of spatial representation in the modeling, with limited efforts for considering the temporal context of urban sprawl. In this paper, we developed a Logistic-Trend-CA model by proposing a trend-adjusted neighborhood as a weighting factor using the information of historical urban sprawl and integrating this factor in the commonly used Logistic-CA model. We applied the developed model in the Beijing-Tianjin-Hebei region of China and analyzed the model performance to the start year, the suitability surface, and the neighborhood size.

Results: Our results indicate the proposed Logistic-Trend-CA model outperforms the traditional Logistic-CA model significantly, resulting in about $18 \%$ and $14 \%$ improvements in modeling urban sprawl at medium (1 km) and fine $(30 \mathrm{~m})$ resolutions, respectively. The proposed Logistic-Trend-CA model is more suitable for urban sprawl modeling over a long temporal interval than the traditional Logistic-CA model. In addition, this new model is not sensitive to the suitability surface calibrated from different periods and spaces, and its performance decreases with the increase of the neighborhood size.
\end{abstract}

Conclusion: The proposed model shows potential for modeling future urban sprawl spanning a long period at regional and global scales.

Keywords: Cellular automata (CA) model, Temporal context, Urban sprawl, Logistic regression, Neighborhood

\section{Introduction}

Urban sprawl modeling is crucial for evaluating potential ecological and environmental risks caused by global urbanization in the future. By 2050, it is expected that the global urban population will reach $\sim 70 \%$ of the world's population (United Nations 2019). Such an explosive growth of urban population would result in the rapid expansion of urban extent, particularly in the fastdeveloping areas such as Asia and Africa. The rapid urban sprawl has many adverse effects on sustainable development, such as air pollution, agriculture land loss, deforestation, and public health (DeFries et al. 2010;

\footnotetext{
* Correspondence: yuyuzhou@iastate.edu

Department of Geological and Atmospheric Sciences, lowa State University, Ames, IA 50011, USA
}

\section{Springer Open}

Foley et al. 2005; Gong et al. 2012; Li et al. 2019b; Zhang et al. 2012). Therefore, to evaluate and minimize these risks and pursue cities' ecological and environmental sustainability, modeling future urban sprawl under different scenarios is highly required for policymakers and scientists to analyze the urban dynamics in complex resource-constrained environments and then make good decisions for city planning and management (Huang et al. 2019; Li and Gong 2016).

Cellular automata (CA)-based models have been widely explored in urban sprawl modeling for simplicity, transparency, and flexibility (Santé et al. 2010). The key of the CA-based urban sprawl model (i.e., urban CA model) is the self-evolution of the urban cell that is driven by its neighbors (Batty and Xie 1994). Thus far, a 
number of CA-based urban sprawl models have been developed and continuously improved through extensive studies, such as the SLEUTH (Slope, Land use, Exclusion, Urban, Transportation, and Hillshade) model (Clarke et al. 1997), the Logistic-CA model (Wu 2002), the Constrained CA (Li and Yeh 2000), the Fuzzy CA (Liu and Phinn 2003), and the Markov CA (Shafizadeh Moghadam and Helbich 2013). By virtue of different approaches to represent the driving factor of urban development by transition rules and neighborhoods, these CA-based urban sprawl models can produce an urban morphology that is close to actual urban lands ( $\mathrm{Li}$ et al. 2017b; Liao et al. 2019). Among these models, the Logistic-CA model is popular because of its easy implementation and explanation.

Transition rules and neighborhoods are two critical components in urban CA models. The original transition rules of urban CA models are explicit "if-then" regulations. These rules were later developed as probabilitybased rules (or called suitability surface) that integrate multiple spatial proxies (Li and Gong 2016; Santé et al. 2010). Different approaches and models, including empirical methods such as regressions and multi-criterion estimation (Hu and Lo 2007; Wu 1998, 2002) and nonlinear models such as decision tree or artificial neural networks (Li and Yeh 2004), have been widely explored to obtain the suitability surface. Although non-linear models (e.g., machine learning approaches) can achieve a better performance than the empirical regression approach, the resulting suitability surface is difficult to explain. For the neighborhood of urban CA models, most studies focus on its spatial configuration (Kocabas and Dragicevic 2006). The shape and size are two common indicators used to form the configuration of the neighborhood. Additional information within the neighborhood, such as the distance to the central cell (van Vliet et al. 2009) and the land use/cover composition (Wu et al. 2012), was also widely studied in the urban CA modeling.

At present, limited efforts have been made to explore the temporal trend of urban sprawl, although many urban CA models have been developed. These developed urban CA models mostly focused on improving the model capacity regarding the spatial information, e.g., the distance of the central cell to surrounding urban infrastructures (Santé et al. 2010; van Vliet et al. 2009). Despite the growth of cities generally follows its historical pathway, the temporal context of urban development was seldom included in the urban CA models. That is, compared with regions developed at early years, the more recently developed regions have a higher likelihood to be developed in the near future (Liu et al. 2017). Satellite observations also confirmed this trend from a long temporal perspective at national and global scales
(Gong et al. 2019; Huang et al. 2020; Li et al. 2015a; Zhou et al. 2018; Zhou et al. 2014; Zhou et al. 2015). Thus, the information of the temporal context of urban development shows a great potential to improve the performance of urban CA models.

Motivated by this idea, in this paper, we firstly developed an improved urban CA model using a trendadjusted neighborhood, of which the historical pathway of urban sprawl was considered. We applied this newly developed urban CA model in the Beijing-Tianjin-Hebei region, a rapidly developing region in the North Plain of China.

\section{Methods}

\section{Study area}

The Beijing-Tianjin-Hebei region, in the north of North China Plain, is the largest urban metropolitan areas of China (Fig. 1). This region occupies an administrative area of $216,600 \mathrm{~km}^{2}$ (Dong et al. 2008), with more than 100 million people. During the past decades, this region experienced rapid growth of population and economy, resulting in a notable sprawl of urban extent. As two primary engines of this region, Beijing and Tianjin lead the development in this area with almost an exponential growth of urban areas over past decades (Chai and $\mathrm{Li}$ 2018; Li et al. 2015a). This rapid urbanization is raising public concerns on water scarcity ( $\mathrm{Li}$ et al. 2018a), energy consumption (Wang and Chen 2016), and air pollution (Liu et al. 2018). Therefore, modeling of historical urban sprawl and predicting of future growth are urgently needed in this region.

\section{Data collection}

We collected seven spatial proxies in the BeijingTianjin-Hebei region in the urban sprawl modeling (Table S1). These proxies consist of spatial features and images such as terrain, land cover, traffic, and location. Except for proxies in the category of terrain (i.e., elevation and slope), all other proxies were processed as the distance to city centers, roads, and specific land cover types (Fig. 2). These spatial proxies were used to determine the suitability of urban sprawl of each pixel, according to their biophysical and socioeconomic conditions (Li et al. 2014).

We used the annual urban extent data derived from nighttime light (NTL) and Landsat satellite data in the model calibration and evaluation. These two annual urban extent datasets were used not only for providing the temporal trend of urban sprawl but also for evaluating the robustness of the improved urban CA model. The NTL-derived urban extent maps (Fig. 2h) span from 1992 to 2013, with a medium resolution of $1 \mathrm{~km}$. The mean accuracy of this developed dataset is about $89 \%$ in China (Zhou et al. 2018). The Landsat-derived urban 


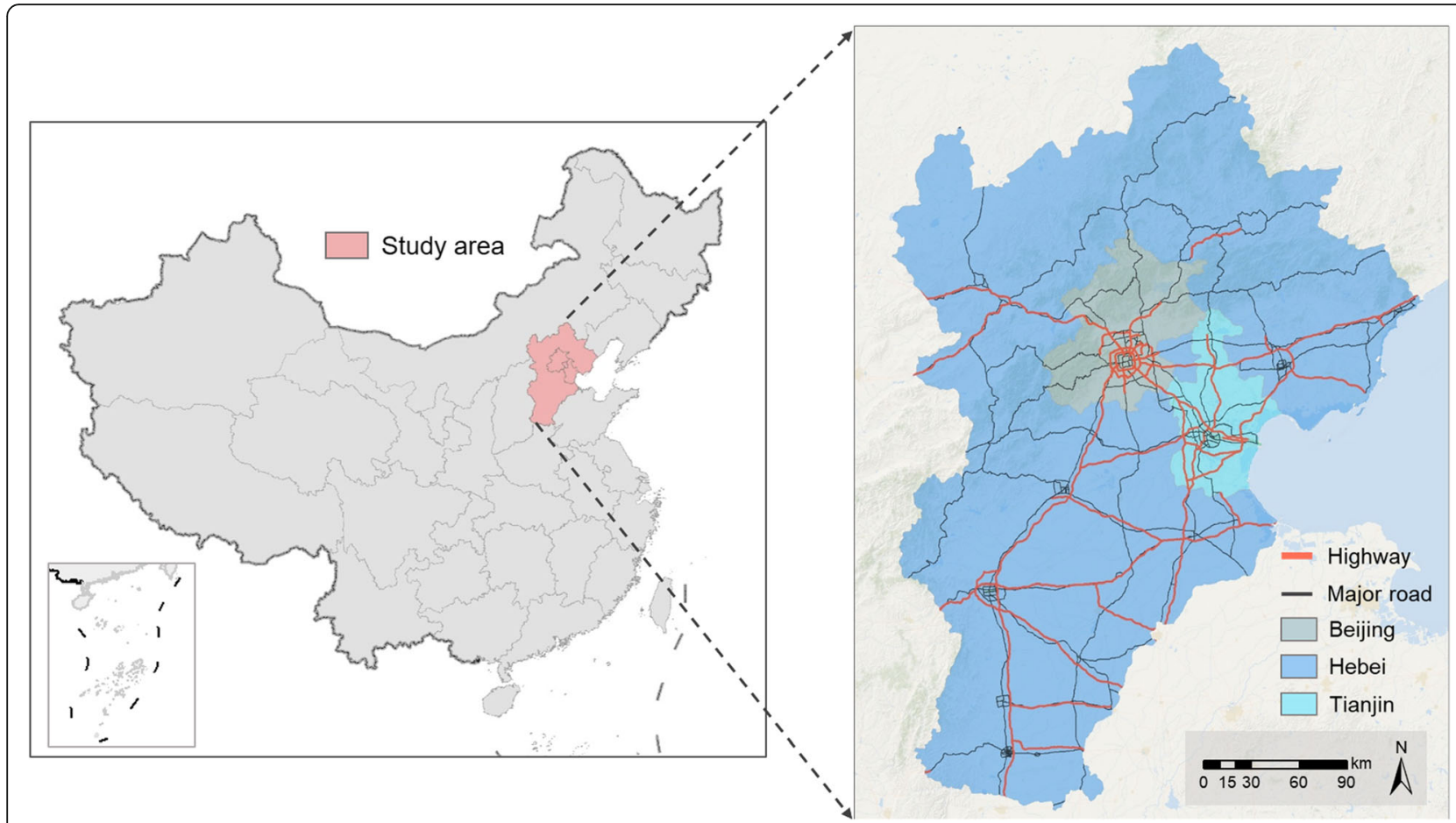

Fig. 1 The geographic location of the Beijing-Tianjin-Hebei region in China
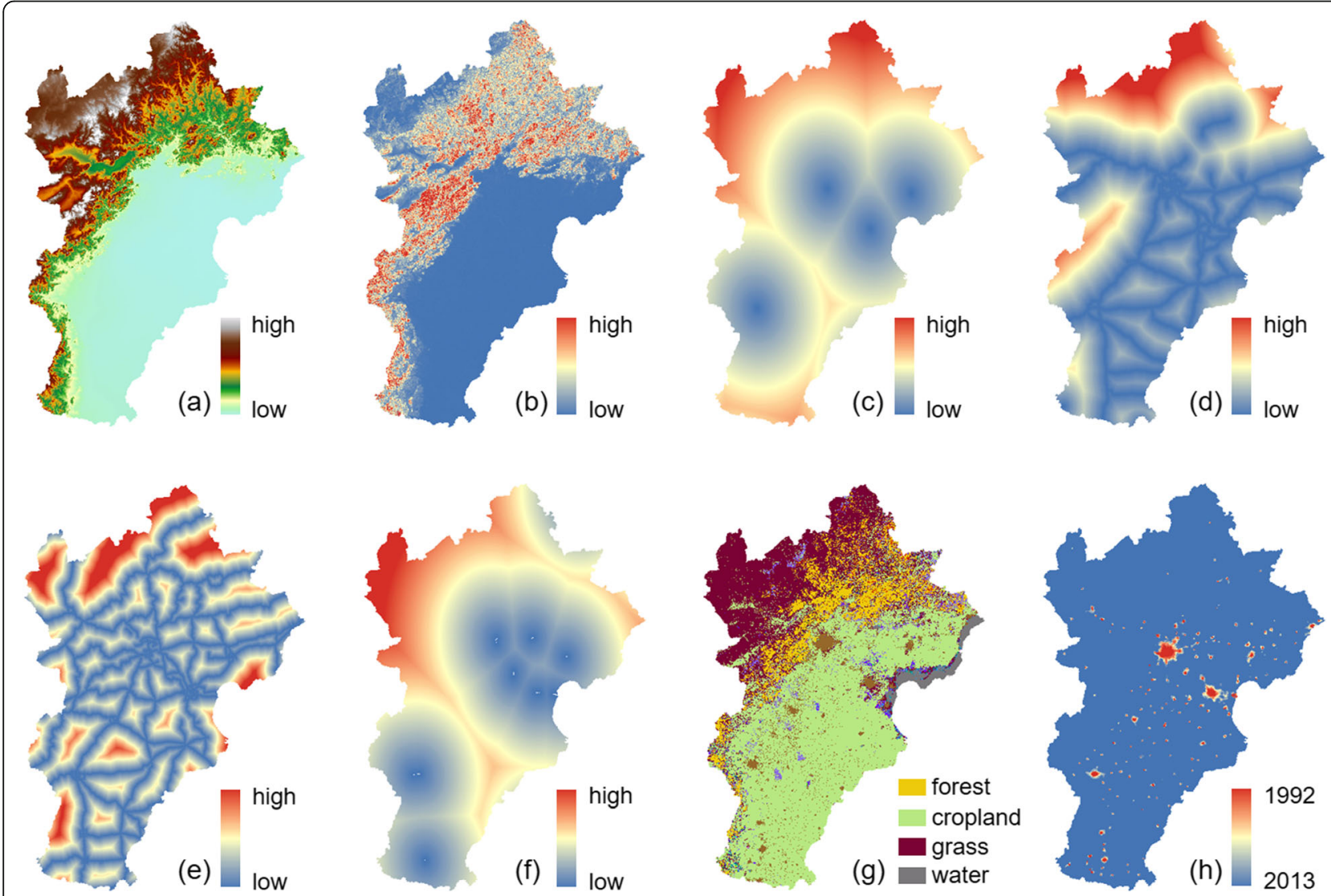

Fig. 2 Spatial proxies and urban extent dynamics in the Beijing-Tianjin-Hebei region. Elevation (a), slope (b), distance to city centers (c), distance to highways (d), distance to major roads (e), distance to local roads (f), land cover $(\mathbf{g})$, and urban extent dynamics from NTL observations (h) 
extent maps (Fig. S1) have a longer temporal interval (1985-2015) than the NTL-derived results, with a fine spatial resolution of $30 \mathrm{~m}$. Based on the long-term Landsat time series data, this Landsat-derived dataset was generated by a temporal segmentation approach (Li et al. 2018b). The overall accuracy of the detected urbanized year in this dataset is around $83 \%$ in the BeijingTianjin-Hebei region. Besides, both of these two urban dynamic datasets follow the logic of urban development. That is, this development is a monotonic conversion from non-urban to urban (Li et al. 2015a).

\section{Framework}

We developed a Logistic-Trend-CA model and assessed its performance to relevant factors in urban CA model (Fig. 3). First, we proposed a trend-adjusted neighborhood with the consideration of the historical pathway of urban sprawl and developed a Logistic-Trend-CA model. Second, we analyzed the performance of the proposed model to key elements in the urban CA model, including the start year of modeling, the suitability surface, and the neighborhood size. The improved urban CA model was applied at $1-\mathrm{km}$ and $30-\mathrm{m}$ spatial resolutions to explore its capability in cross-scale modeling. Details of each step are given in the following sections.

\section{The logistic-trend-CA model}

The urban CA model is a grid-based self-evolution system to simulate the dynamics of urban land (Batty and Xie 1999). In this system, the status (i.e., urban and nonurban) of each grid is determined by its surrounding neighbors. A non-urban grid is more likely to change to urban in the near future if there are more urban grids surrounded. Evolution of massive grids using this rule simultaneously can simulate the change of complex urban landscapes. With the consideration of additional spatial factors such as traffic networks and land covers, the urban CA model can be used to simulate the dynamic of urban land with a high degree of reliability.

We built our Logistic-Trend-CA model on the Logistic-CA model as it has been widely used in urban sprawl modeling due to its clear explanation of spatial proxies and ease of implementation ( $\mathrm{Hu}$ and Lo 2007; $\mathrm{Wu}$ 2002). The logistic regression function is the key of the Logistic-CA model. Its output is a spatially explicit suitability surface, which indicates the suitability for development under considerations of different spatial proxies. Assuming there are $n$ spatial proxies $\left[x_{1}, x_{2}, \ldots\right.$ $\left.x_{n}\right]$, the logistic regression function can be expressed as Eqs. (1 and 2).

$$
\begin{aligned}
& z=b_{1} x_{1}+\cdots+b_{n} x_{n} \\
& P_{\text {suit }}=1 /\left(1+\exp ^{-z}\right)
\end{aligned}
$$

where $P_{\text {suit }}$ is the obtained suitability of development from the biophysical and socioeconomic conditions and $b_{i}$ and $x_{i}$ are the $i$ th coefficient and spatial proxy, respectively.

We improved the neighborhood of the Logistic-CA model by considering the historical pathway of urban sprawl. The neighborhood is a crucial component in the urban CA model because it is a basic driver of urban dynamics modeling (Kocabas and Dragicevic 2006). The configuration of the neighborhood is closely related to its size, shape, and surrounding land cover types. Here, we developed the trend-adjusted neighborhood by incorporating the historical pathway of urban sprawl as a weighting factor, based on the widely used Moore configuration (Eqs. (3 and 4)).

$$
\begin{aligned}
& W_{i j}^{t s}=1-\frac{N_{i j}^{u}}{N} \\
& \Omega=\frac{\sum_{m \times m} \operatorname{Con}\left(S_{i j}=\text { urban }\right) \times W_{i j}^{t s}}{m \times m-1}
\end{aligned}
$$

where $\Omega$ is the influence of neighborhood that considers the historical pathway of urban sprawl using a weighting factor of $W_{i j}^{t s} . N_{i j}^{u}$ is the accumulated year of cell $(i, j)$ with the status as urban from the annual urban time series data with a temporal interval of $N$. As a result, for

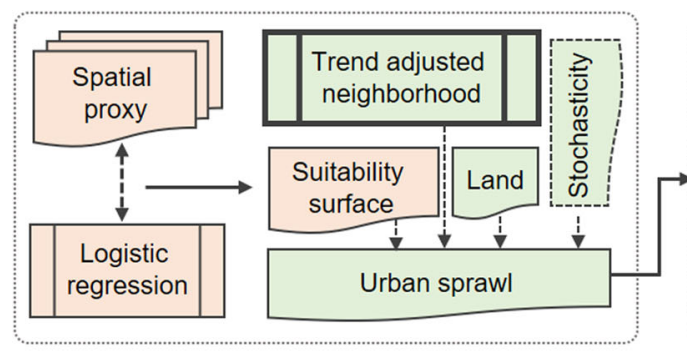

(a) Logistic-Trend-CA model

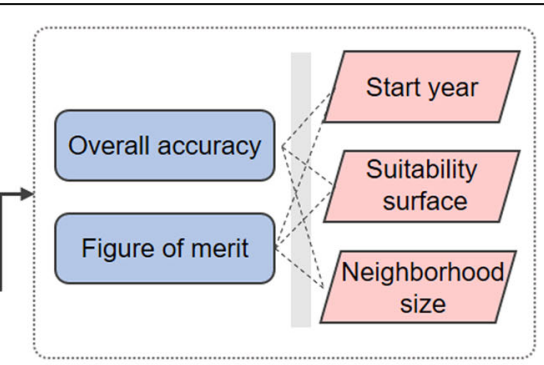

(b) Validation of model performance

Fig. 3 The framework of the Logistic-Trend-CA model (a) and the validation of model performance to key factors (b) 
a potential cell, urban neighbors that were developed in more recent years have larger impacts than those developed in earlier years. $m$ is the window size, and Con() is a conditional function, which returns 1 when the status of cell $(i, j)$ is urban.

Compared with traditional neighborhoods, the developed trend-adjusted neighborhood can result in a sprawl pattern following the historical pathway, as illustrated in Fig. 4. Urban sprawl has an inertia of development as it generally follows the temporal trend of historical development (Liu et al. 2017), i.e., there is a relatively higher development probability around those newly developed urban areas. As a result, the weighting factors of $W_{i j}^{t s}$ of urban pixels developed in more recent years are higher than those developed in earlier years. Assuming the weighting factors of urbanized pixels across years are different as illustrated in Fig. 4 (a), thus, pixels 1 and 2 have the same neighborhood influence if using the traditional neighborhood. However, if taking into account of the historical pathway of urban sprawl, pixel 2 has a higher neighborhood influence because its surrounding pixels were developed more recent compared to pixel 1 (Fig. 4 (b)), although they have the same number of urban neighbors for pixels 1 and 2. Such smaller difference regarding the neighborhood influence would result in a notable different sprawl pattern after several iterations (Fig. 4 (c)), as most urbanized pixels were developed following the historical pathway if the neighborhood was adjusted by the temporal trend.

We also included land constraint and stochastic perturbation in the developed Logistic-Trend-CA model. Restricted lands, such as water and protected areas, were not considered for development in our model; thus, they were represented as a land constraint term as Land $=0$ ( $\mathrm{Li}$ et al. 2014). In addition, we used the stochastic perturbation $S P$ to represent unconsidered factors (e.g., policy) in the modeling (White and Engelen 1993), as expressed in Eq. (5).

$$
S P=1+(-\ln \lambda)^{\alpha}
$$

where $S P$ is the stochastic perturbation, $\lambda$ is a random value $[0,1]$, and $\alpha$ is a parameter to determine the degree of perturbation.

The development probability was calculated based on the suitability surface, neighborhood, land constrain, and stochastic perturbation. For urban time series data derived from NTL and Landsat, we determined their

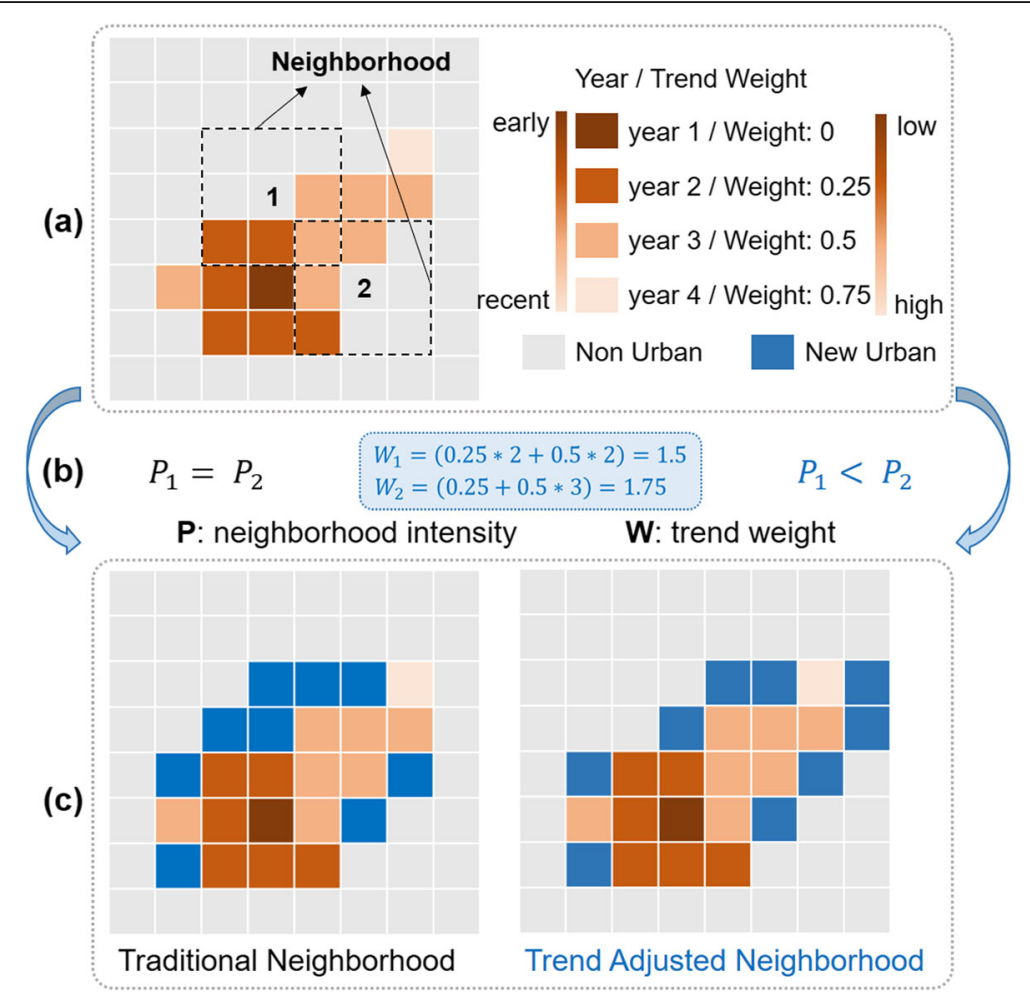

Fig. 4 Illustration of the trend-adjusted neighborhood in the urban CA model. Urban pixels with different developing years and weights (a), calculation of neighborhood impacts for pixels 1 and 2 (b), and modeled urban sprawl using different neighborhoods (c). This illustrative figure shows the results of allocating three urban pixels per iteration, with a total of nine urban pixels. Trend weights in (a) were calculated using Eq. (3) with a temporal span of 4 years 
development probabilities using Eqs. (6) and (7), respectively. The $S P$ is not considered in the modeling with NTLderived urban extent maps, due to the homogeneity of urban land within the boundary (Zhou et al. 2018).

$$
\begin{aligned}
& P_{\text {dev }}=P_{\text {suit }} \times \Omega \times \text { Land } \\
& P_{\text {dev }}=P_{\text {suit }} \times \Omega \times \text { Land } \times S P
\end{aligned}
$$

\section{Model validation}

We assessed the performance of the developed LogisticTrend-CA model to key factors in urban CA model. Two quantitative metrics were used for the assessment, namely the overall accuracy (OA) and the figure of merit (FOM). The OA was directly calculated as the percentage of consistent pixels to all pixels in the entire map, while the FOM indicates the consistency between modeled and observed maps on those changed pixels. The FOM has been widely used in many studies of urban CA models since it can provide a relatively comprehensive evaluation of the model performance (Chen et al. 2014; $\mathrm{Li}$ et al. 2014; Pontius et al. 2007). The FOM can be expressed as Eq. (8) (Pontius et al. 2008).

$$
\mathrm{FOM}=B /(A+B+C) \times 100 \%
$$

where FOM is the figure of merit, $B$ is the number of observed urban pixels that were simulated as urban, $A$ is the number of observed urban pixels that were simulated as non-urban, and $C$ is the number of observed non-urban pixels that were simulated as urban.

We evaluated the model performance by exploring sensitivities of three key factors in our urban CA model: the start year of modeling, the suitability surface, and the neighborhood size. The influences of these three key factors can be quantitatively evaluated with clear physical meanings in the CA model. Although there are other factors that may also influence the model performance, they are considered in the selected elements in the model. For example, the urban spatial configuration can be captured by the neighborhood and stochastic disturbance in the CA model. Other factors (e.g., the degree of urban development) are more related to regional urban demand compared to the spatial allocation of increased urban demand (Li et al. 2019a). First, we examined the modeling capability of the Logistic-Trend-CA model over a long temporal span through changing the start year of modeling, which is closely related to the iterations and error propagation in the modeling ( $\mathrm{Li}$ et al. 2014). Second, we investigated the model performance to suitability surfaces using calibrated results from different periods. The suitability surface characterizes the likelihood of urban development from the biogeophysical (e.g., terrain and land cover) and socioeconomic (e.g., traffic networks) aspects. In addition, we evaluated the derived suitability surface using the receiver operating characteristic (ROC) approach (Liu et al. 2017; Pontius Jr et al. 2001; Wu et al. 2009). The ROC curve was calculated by dividing the continuous suitability surface into binary maps using different thresholds and then comparing the derived binary map with the reference map. Finally, we compared the model performance by varying neighborhood sizes. The neighborhood is a crucial component to drive the self-evolution of urban land system, and the neighborhood size reflects the degree of local impact from neighbors (Kocabas and Dragicevic 2006). Many urban CA models have been developed for particular applications with different structures, functions, and data requirements ( $\mathrm{Li}$ and Gong 2016). Quantitative indicators such as the FOM have been used for comparing urban CA models. We evaluated our model performance based on the FOM and compared it with previous studies. In addition, we compared our model with the similar Logistic-CA model, which has been widely used in previous urban CA studies and can serve as a benchmarking model, for several key factors. The Logistic-CA model also has the same structure as our proposed model except for the consideration of the neighborhood.

\section{Setting of the urban CA model}

The inputs of urban CA model are the urban extent map in the beginning year associated with a variety of spatial proxies (Fig. 2) and a set of parameters (Li et al. 2017a), and the output of our model is the urban extent map in the target year. In our study, the neighborhood size $(m)$ was set as 3 and 5 in calculating the influence of neighborhood $(\Omega)$ using Eq. (4), for urban sprawl modeling with medium $(1 \mathrm{~km})$ and fine $(30 \mathrm{~m})$ resolutions, respectively. The degree of stochastic perturbation $(\alpha)$ was set as 3 as suggested for modeling at a $30-\mathrm{m}$ resolution using Eq. (5) (Li et al., 2014). Also, we set the restricted conversion type as water in our study to avoid the conversion from water to urban. Finally, the modeled results were compared with the observed urban extent map from remote sensing observations in the same year.

\section{Results and discussion}

\section{Performance of the logistic-trend-CA model}

The developed Logistic-Trend-CA model using the trend-adjusted neighborhood outperformed the traditional Logistic-CA model. Improvements of FOM are about $18 \%$ and $14 \%$ for urban sprawl modeling at medium $(1 \mathrm{~km})$ and fine $(30 \mathrm{~m})$ resolutions, respectively (Figs. 5 and 6). The OA increases by around $2-3 \%$ using the Logistic-Trend-CA model. This suggests the developed model is robust regarding the model performance at different resolutions. Specifically, the omission (i.e., pixels observed as 
urban but simulated as non-urban) and commission (i.e., pixels observed as non-urban but simulated as urban) errors were considerably reduced when the historical pathway of urban sprawl was considered (Figs. 5 and 6). That is, the trend-adjusted neighborhood improves the urban sprawl pathway during the modeling, which further reduces the error generation and propagation ( $\mathrm{Li}$ et al. 2014; Yeh and $\mathrm{Li}$ 2006), particularly for modeling over a relatively long temporal interval. As a result, the developed LogisticTrend-CA model can simulate more realistic urban forms (or landscapes) compared to the traditional Logistic-Trend-CA model. However, it is worth to note that the improvement of Logistic-Trend-CA model is related to the time span of the used temporal information in the neighborhood. The improvement of FOM in Fig. 5 would decrease to 10\% and $6 \%$ when the time span reduces to 10 and 5 years, respectively. If using a very limited time span (e.g., 1-2 years), outputs from Logistic-Trend-CA and Logistic-CA models are almost the same.

\section{Validation of model performance to key factors The start year of modeling}

A shorter modeling period with the start year of 2002 can increase the OA but decrease the FOM of the modeled result compared to a longer modeling period with the start year of 1992. Since the time step of our modeling is annual, the impact of start year on the model performance is mainly determined by the number of iterations, which further affect the error generation and propagation in the modeling ( $\mathrm{Li}$ et al. 2014). The comparison of model performance using different temporal intervals indicates an opposite trend of OA and FOM (Fig. 7 and Fig. S2). That is, the OA is slightly increased from 97 to $98 \%$ while the FOM is decreased from 52 to $44 \%$ when using a relatively shorter modeling period at a medium resolution $(1 \mathrm{~km})$. Similarly, for urban extent maps with a fine resolution $(30 \mathrm{~m})$, the improvement of $\mathrm{OA}$ is about $2 \%$ while the decrease of FOM is about $8-9 \%$. Such a contrastive trend of $\mathrm{OA}$ and $\mathrm{FOM}$ is related to the consistency of urban extent maps between the start and end years. For example, although the FOM of modeling with a start year of 2002 (Fig. 7b) is lower than that with a start year of 1992 (Fig. 7a), the initial urban extent in 2002 has excluded errors generated and propagated from 1992 to 2002, resulting in a higher overall agreement.

The improvement of the Logistic-Trend-CA model relative to the Logistic-CA model decreases when modeling with a relatively short period (Fig. 8). Introducing the historical pathway of urban sprawl improves the performance using the Logistic-Trend-CA

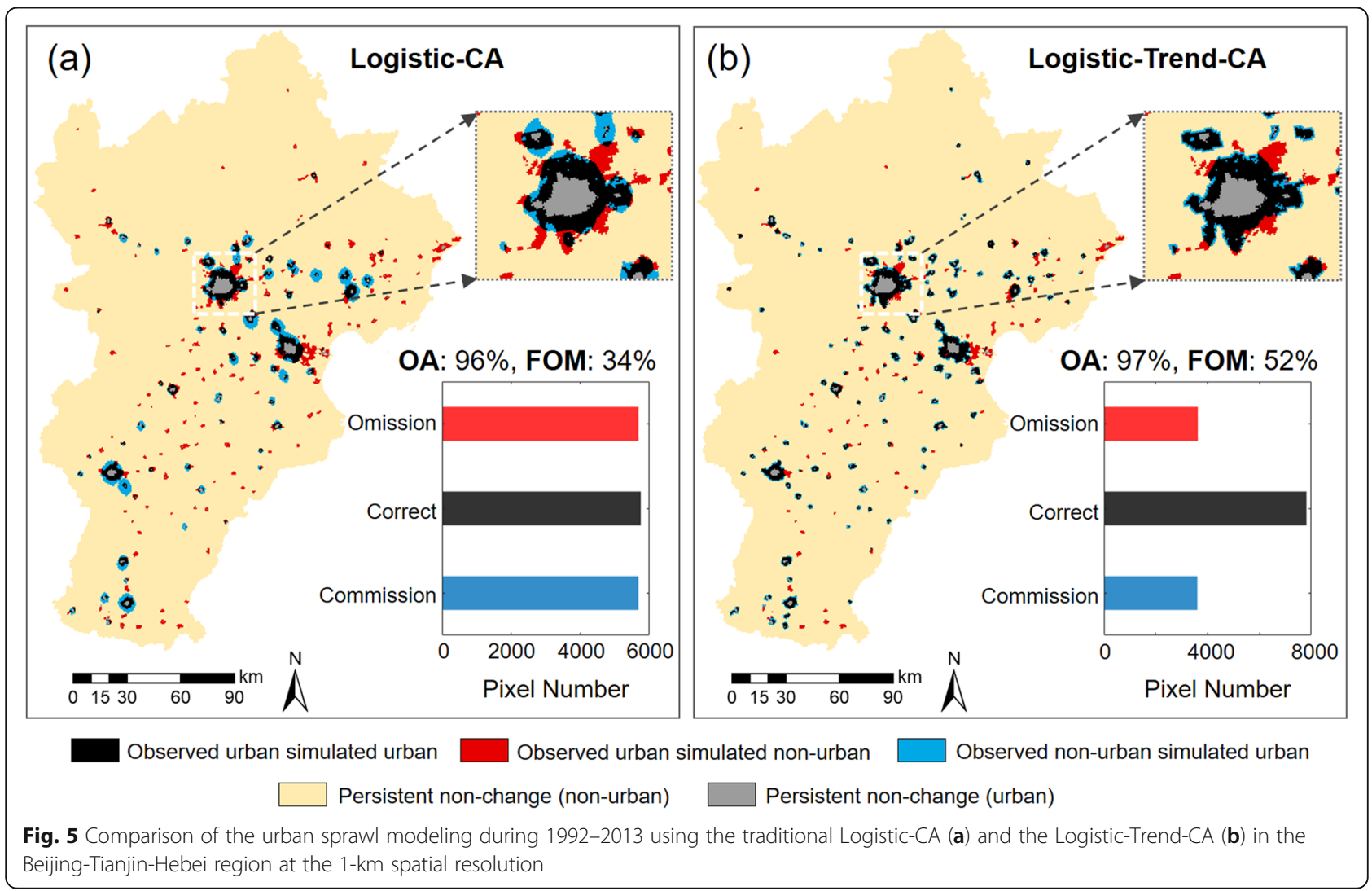




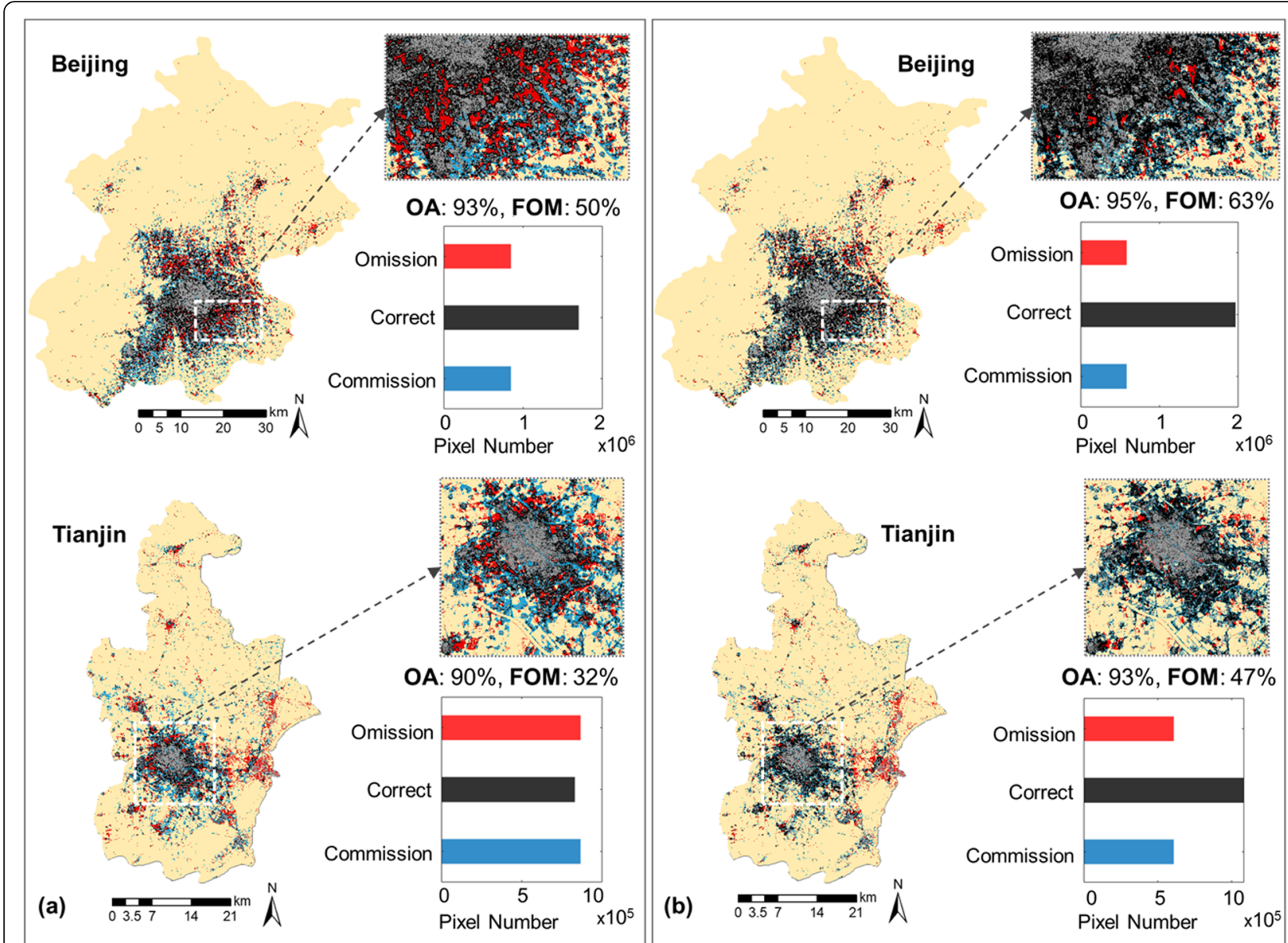

Observed urban simulated urban

Observed urban simulated non-urban

Observed non-urban simulated urban

Persistent non-change (non-urban)

Persistent non-change (urban)

Fig. 6 Comparison of the urban sprawl modeling during 1985-2015 using the traditional Logistic-CA (a) and the Logistic-Trend-CA (b) in Beijing and Tianjin at a 30-m spatial resolution

model in areas with similar neighborhood influences and development suitability. Our results indicate the FOM is improved from 34 to $44 \%$ in the BeijingTianjin-Hebei region at a medium resolution $(1 \mathrm{~km})$, when the modeling period is shortened from 19922013 to 2002-2013 (Fig. 8a). Similar conclusions are also confirmed in modeling cases of Beijing and Tianjin at the $30-\mathrm{m}$ spatial resolution during the modeling period of $2005-2015$, i.e., the FOM is increased from 53 to $54 \%$ in Beijing and from 32 to $39 \%$ in Tianjin (Fig. 8b, c). Although the improvement of FOM is reduced by about $8 \%$ when the modeling period is shortened from 1992-2013 (Fig. 5) to 2002-2013 (Fig. 8a) in the Beijing-TianjinHebei region, the Logistic-Trend-CA model remains a good performance compared to the Logistic-CA model, particularly for modeling cases with relatively worse performances of the suitability surface (i.e.,
Tianjin) (Fig. 9c). Also, it should be noted that the performance of the proposed model is related to urban expansion patterns in cities, and the improvement of the Logistic-Trend-CA model for a concentric growth across different directions around the urban center is not significant (e.g., Fig. 8b).

\section{The suitability surface}

Our Logistic-Trend-CA model is not sensitive to suitability surfaces that are derived during different periods. These suitability surfaces were calibrated using the logistic regression model, based on training samples collected from urbanized and persistent regions in different periods. We found the temporal effect of training samples collected from different periods on the derived suitability surface is limited (Fig. 9), i.e., their ROC curves are close. The impact of different suitability surfaces on urban sprawl modeling is 


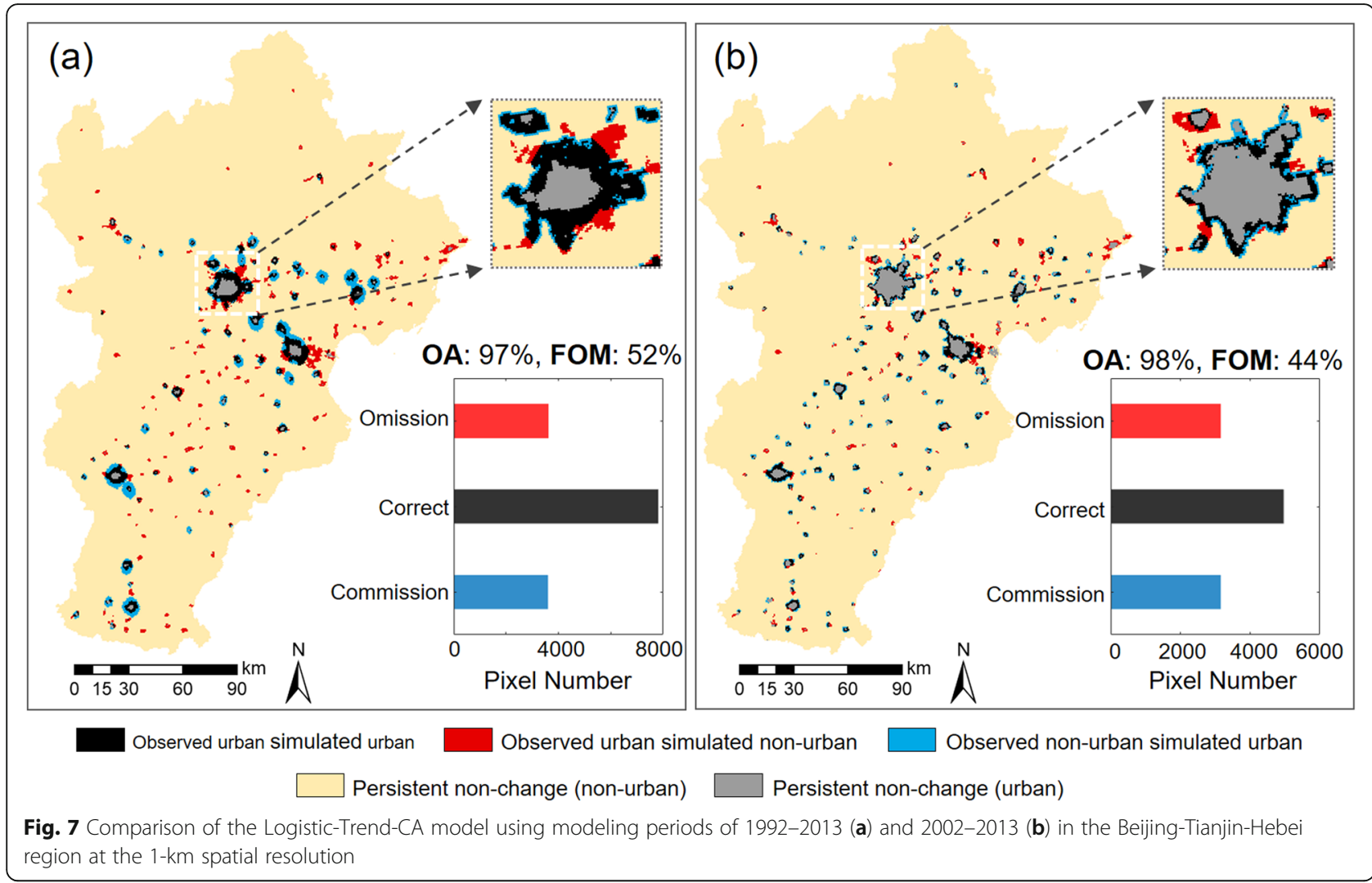

considerably reduced with the increase of iterations during the simulation (Table 1), i.e., the FOMs are similar in these experiments. Modeled results at both the $1-\mathrm{km}$ and $30-\mathrm{m}$ spatial resolutions are almost the same using suitability surfaces in different periods. This is because the development probability of urban sprawl in each iteration is jointly determined by the neighborhood and the suitability surface. The suitability surface is assumed to be persistent, and its contribution is reduced as errors propagated during the modeling (Li et al. 2015b; Santé et al. 2010), whereas the trend-adjusted neighborhood is updated iteratively and plays a dominant role in the modeling. It should be noted that suitability surfaces used in our study were mainly derived from the logistic regression model, which is a statistical model and is more robust for training samples compared with data miningbased approaches such as random forest and neural networks (Li et al. 2014).

\section{The neighborhood size}

The performance of Logistic-Trend-CA decreases with the increase of the neighborhood size (Fig. 10). This finding is consistent for urban sprawl modeling at different spatial resolutions (i.e., $1 \mathrm{~km}$ and $30 \mathrm{~m}$ ). The FOM is highest when the neighborhood size is 3 ; thereafter, it decreases with the increase of the neighborhood size. This relationship is more distinctive when the window size is small (e.g., lower than 7), suggesting the contribution of included weighting factors from the historical pathway of urban sprawl decreased when increasing the neighborhood size (Eq. 4). Accordingly, the improvement of the Logistic-Trend-CA model relative to the Logistic-CA model was reduced. In addition, urban sprawl modeling is more sensitive to the neighborhood size with a relatively coarse spatial resolution, comparing modeled results at the 1-km (Fig. 10a) and 30-m (Fig. 10b, c) spatial resolutions. The impact of local neighbors on the model performance decreases due to the decrease of neighborhood intensity of the central pixel, with the increase of neighborhood size.

\section{Conclusions}

In this study, we developed a Logistic-Trend-CA model with the consideration of the historical pathway of urban sprawl and tested it in the Beijing-Tianjin-Hebei region of China. In this model, we proposed a trend-adjusted neighborhood as a weighting factor using the historical pathway of urban sprawl. This improved neighborhood was integrated with the widely used logistic regression function to simulate urban sprawl. We applied this model in the Beijing-Tianjin-Hebei region using the time 

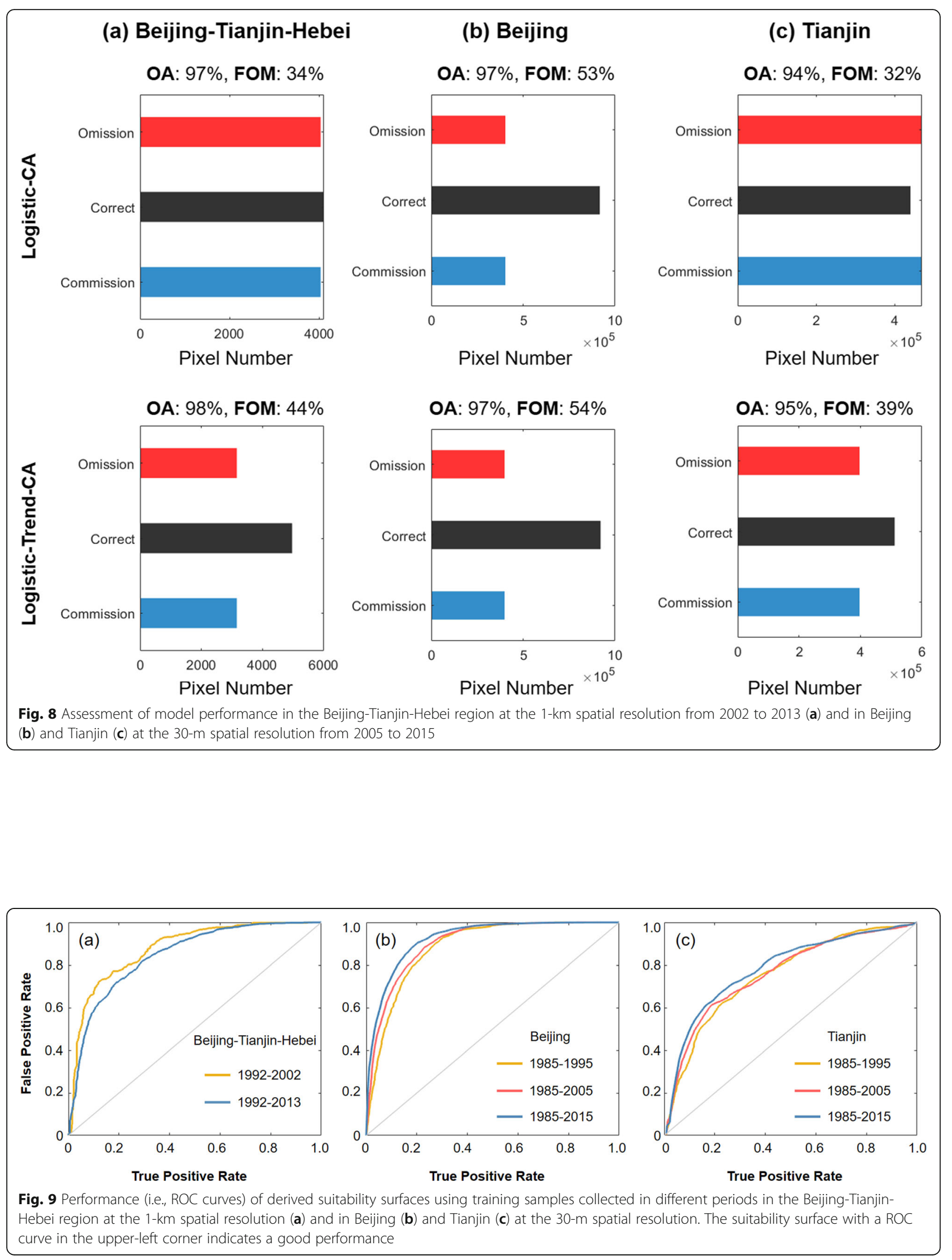
Table 1 Assessment of the urban sprawl modeling using suitability surfaces derived from different periods

\begin{tabular}{|c|c|c|c|c|c|c|}
\hline \multirow[b]{2}{*}{ Suitability surface } & \multicolumn{2}{|c|}{ Beijing-Tianjin-Hebei (1 km) } & \multicolumn{2}{|c|}{ Beijing (30 m) } & \multicolumn{2}{|c|}{ Tianjin (30 m) } \\
\hline & 1992-2002 & 1992-2013 & 1985-1995 & $1985-2015$ & 1985-1995 & $1985-2015$ \\
\hline $\mathrm{OA}(\%)$ & 97.4 & 97.4 & 95.4 & 95.5 & 93.3 & 93.3 \\
\hline FOM (\%) & 52.1 & 52.0 & 62.7 & 62.7 & 46.7 & 47.1 \\
\hline
\end{tabular}

series data of urban extent from NTL and Landsat observations. Model performance was evaluated and compared with the traditional Logistic-CA model. The robustness was explored through analyzing the model performance to key factors in urban CA model.

We found our Logistic-Trend-CA model notably outperforms the traditional Logistic-CA model. The improvement of FOM is around $18 \%$ and $14 \%$ using the Logistic-Trend-CA model at the $1-\mathrm{km}$ and $30-\mathrm{m}$ spatial resolutions, respectively, compared to the traditional Logistic-CA model. The Logistic-Trend-CA model performs well for modeling studies with a long temporal span. In addition, it is not sensitive to suitability surfaces derived from the logistic regression model in different periods, and the trend-adjusted neighborhood plays an important role in the modeling. Finally, the performance of the Logistic-TrendCA model decreases with the increase of neighborhood size.

This study opens a new research avenue to incorporate the temporal context information in urban CA models. Through using the temporal context information of historical urban sprawl, the LogisticTrend-CA model shows a good performance in simulating future urban sprawl with a long interval (e.g., decades). Also, the developed urban CA model in this study performs well at the $1-\mathrm{km}$ spatial resolution, showing its capabilities in global urban sprawl modeling using the time series data of urban extent from NTL observations (Zhou et al. 2018). Thus, our developed urban CA model can be used for urban expansion modeling with a long temporal span because historical growth of urban extent can be incorporated into the modeling. Such improvement can mitigate the uncertainty in modeling urban growth using the information of temporal contexts. However, it is worth to note that the temporal effect of suitability surface (e.g., road expansion and land cover change during the modeling period) is not considered in our model, although we included the dynamics of urban extent in the neighborhood component. Also, our model needs improvement for simulating policy-induced changes in urbanized areas without neighboring urban pixels (Liu et al. 2010), which could occur in rapidly developing regions in China. The corresponding improvements for these common limitations in CA models are needed for our Logistic-Trend-CA model in future studies.

\section{Supplementary information}

Supplementary information accompanies this paper at https://doi.org/10 1186/s13717-020-00234-9.

Additional file 1: Fig. S1. Urban extent dynamics from Landsat observations. Fig. S2. Comparison of the Logistic-Trend-CA model using modeling periods of 1985-2015 (a) and 2005-2015 (b) in Beijing and Tianjin at the 30-m spatial resolution. Table S1. Spatial proxies used in the modeling.

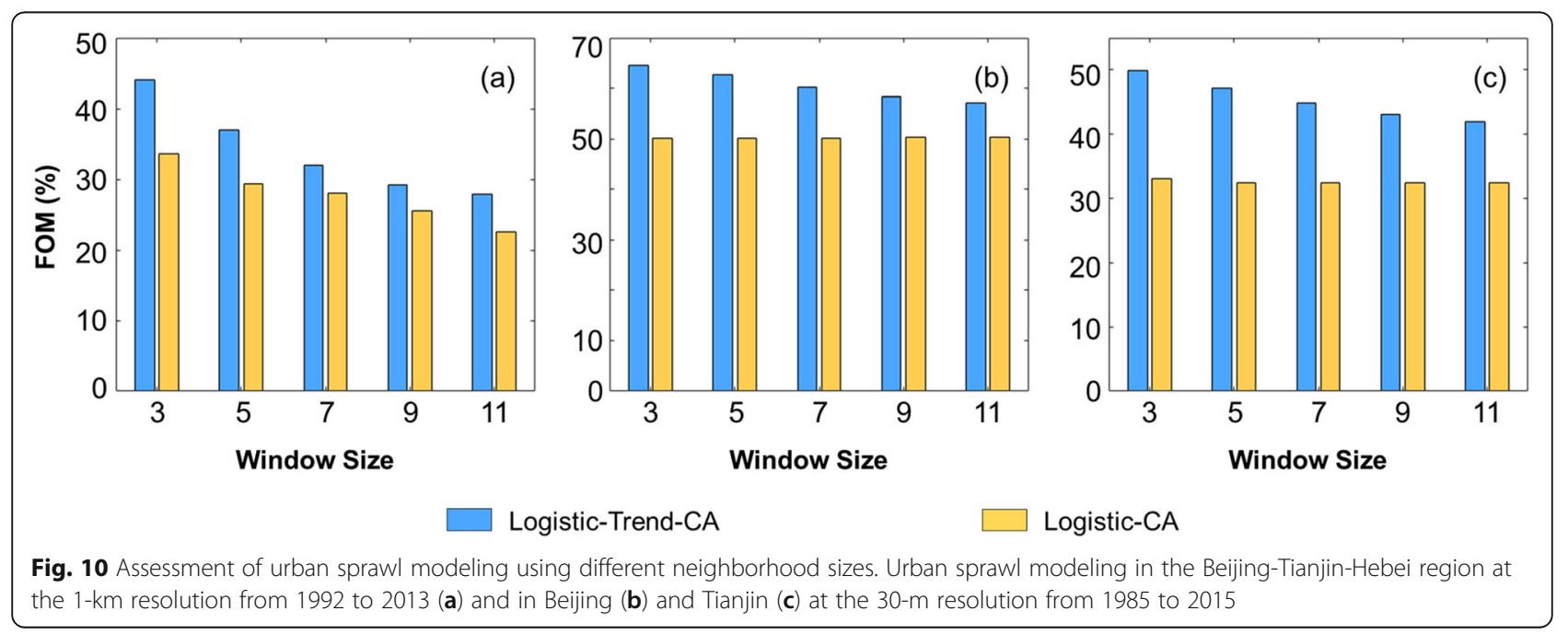




\section{Abbreviations}

CA: Cellular automata; FOM: Figure of merit; ROC: Receiver operating characteristic

\section{Acknowledgements}

Not applicable.

\section{Authors' contributions}

Y.Z. designed the work; X.L. performed the analysis; X.L. and Y.Z. drafted the paper; and W.C. contributed to the interpretation of the results and to the writing of the paper. The author(s) read and approved the final manuscript.

\section{Funding}

This study was supported by the National Science Foundation (CBET1803920).

\section{Availability of data and materials}

The datasets used and/or analyzed during the current study are available from the corresponding author on reasonable request.

\section{Ethics approval and consent to participate} Not applicable.

\section{Consent for publication}

Not applicable.

\section{Competing interests}

The authors declare that they have no competing interest.

Received: 4 March 2020 Accepted: 17 May 2020

\section{Published online: 29 May 2020}

\section{References}

Batty M, Xie Y (1994) From cells to cities. Environ Plan B 21(7):31-48

Batty M, Xie Y (1999) Self-organized criticality and urban development. Discret Dyn Nat Soc 3(2-3):109-124

Chai B, Li P (2018) Annual urban expansion extraction and spatio-temporal analysis using Landsat time series data: a case study of Tianjin, China. IEEE J Select Topics Appl Earth Observ Remote Sensing 11(8):2644-2656

Chen Y, Li X, Liu X, Ai B (2014) Modeling urban land-use dynamics in a fast developing city using the modified logistic cellular automaton with a patchbased simulation strategy. Int J Geogr Inf Sci 28(2):234-255

Clarke K, Hoppen S, Gaydos L (1997) A self-modifying cellular automaton mode of historical. Environ Plan B 24:247-261

DeFries RS, Rudel T, Uriarte M, Hansen M (2010) Deforestation driven by urban population growth and agricultural trade in the twenty-first century. Nat Geosci 3(3):178-181

Dong J, Zhuang D, Xu X, Ying L (2008) Integrated evaluation of urban development suitability based on remote sensing and GIS techniques-a case study in Jingjinji area, China. Sensors 8(9):5975-5986

Foley JA, DeFries R, Asner GP et al (2005) Global consequences of land use. Science 309(5734):570-574

Gong P, Li X, Zhang W (2019) 40-year (1978-2017) human settlement changes in China reflected by impervious surfaces from satellite remote sensing. Sci Bull 64(11):756-763

Gong P, Liang S, Carlton EJ et al (2012) Urbanisation and health in China. Lancet 379(9818):843-852

Hu Z, Lo CP (2007) Modeling urban growth in Atlanta using logistic regression. Comput Environ Urban 31(6):667-688

Huang X, Wang Y, Li J et al (2020) High-resolution urban land-cover mapping and landscape analysis of the 42 major cities in China using ZY-3 satellite images Sci Bull https://doi.org/10.1016/j.scib.2020.03.003

Huang X, Xia J, Xiao R, He T (2019) Urban expansion patterns of 291 Chinese cities, 1990-2015. Int J Digital Earth 12(1):62-77

Kocabas V, Dragicevic S (2006) Assessing cellular automata model behaviour using a sensitivity analysis approach. Comput Environ Urban 30(6):921-953

Li S, Yang H, Lacayo M, Liu J, Lei G (2018a) Impacts of land-use and land-cover changes on water yield: a case study in Jing-Jin-Ji, China. Sustainability 10(4): 960

Li X, Gong P (2016) Urban growth models: progress and perspective. Sci Bull 61(21):1637-1650
Li X, Gong P, Liang L (2015a) A 30-year (1984-2013) record of annual urban dynamics of Beijing City derived from Landsat data. Remote Sens Environ 166:78-90

Li X, Gong P, Yu L, Hu T (2017a) A segment derived patch-based logistic cellular automata for urban growth modeling with heuristic rules. Comput Environ Urban 65:140-149

Li X, Liu X, Gong P (2015b) Integrating ensemble-urban cellular automata model with an uncertainty map to improve the performance of a single model. Int J Geogr Inf Sci 29(5):762-785

Li X, Liu X, Yu L (2014) A systematic sensitivity analysis of constrained cellular automata model for urban growth simulation based on different transition rules. Int J Geogr Inf Sci 28(7):1317-1335

Li X, Lu H, Zhou Y et al (2017b) Exploring the performance of spatio-temporal assimilation in an urban cellular automata model. Int J Geogr Inf Sci 31(11): 2195-2215

Li X, Yeh AG-O (2000) Modelling sustainable urban development by the integration of constrained cellular automata and GIS. Int J Geogr Inf Sci 14(2): 131-152

Li X, Yeh G-O (2004) Data mining of cellular automata's transition rules. Int J Geogr Inf Sci 18(8):723-744

Li X, Zhou Y, Eom J, Yu S, Asrar GR (2019a) Projecting global urban area growth through 2100 based on historical time-series data and future shared socioeconomic pathways. Earth's Future 7:351-362

Li X, Zhou Y, Meng L, Asrar GR, Lu C, Wu Q (2019b) A dataset of 30 m annual vegetation phenology indicators (1985-2015) in urban areas of the conterminous United States. Earth System Sci Data 11(2):881-894

Li X, Zhou Y, Zhu Z, Liang L, Yu B, Cao w (2018b) Mapping annual urban dynamics (1985-2015) using time series of Landsat data. Remote Sens Environ 216:674-683

Liao J, Shao G, Wang C, Tang L, Huang Q, Qiu Q (2019) Urban sprawl scenario simulations based on cellular automata and ordered weighted averaging ecological constraints. Ecol Indic 107:105572

Liu H, Fang C, Huang J et al (2018) The spatial temporal characteristics and influencing factors of air pollution in Beijing-Tianjin-Hebei urban agglomeration. J Geogr Sci 73:177-191

Liu X, Li X, Chen Y, Tan Z, Li S, Ai B (2010) A new landscape index for quantifying urban expansion using multi-temporal remotely sensed data. Landsc Ecol 25(5):671-682

Liu X, Liang X, Li X et al (2017) A future land use simulation model (FLUS) for simulating multiple land use scenarios by coupling human and natural effects. Landscape Urban Plan 168:94-116

Liu Y, Phinn SR (2003) Modelling urban development with cellular automata incorporating fuzzy-set approaches. Comput Environ Urban 27(6):637-658

Pontius R Jr, Boersma W, Castella J-C et al (2008) Comparing the input, output, and validation maps for several models of land change. Ann Reg Sci 42(1): $11-37$

Pontius RG Jr, Cornell JD, Hall CAS (2001) Modeling the spatial pattern of landuse change with GEOMOD2: application and validation for Costa Rica. Agric Ecosyst Environ 85(1-3):191-203

Pontius RG, Walker R, Yao-Kumah R et al (2007) Accuracy assessment for a simulation model of Amazonian deforestation. Ann Assoc Am Geogr 97(4): 677-695

Santé I, García AM, Miranda D, Crecente R (2010) Cellular automata models for the simulation of real-world urban processes: a review and analysis. Landscape Urban Plan 96(2):108-122

Shafizadeh Moghadam H, Helbich M (2013) Spatiotemporal urbanization processes in the megacity of Mumbai, India: a Markov chains-cellular automata urban growth model. Appl Geogr 40:140-149

United Nations (2019) World urbanization prospects: the 2018 revision. UN

van Vliet J, White R, Dragicevic S (2009) Modeling urban growth using a variable grid cellular automaton. Comput Environ Urban 33(1):35-43

Wang S, Chen B (2016) Energy-water nexus of urban agglomeration based on multiregional input-output tables and ecological network analysis: a case study of the Beijing-Tianjin-Hebei region. Appl Energy 178:773-783

White R, Engelen G (1993) Cellular automata and fractal urban form: a cellular modelling approach to the evolution of urban land-use patterns. Environ Plann A 25(8):1175-1199

Wu B, Huang B, Fung T (2009) Projection of land use change patterns using kernel logistic regression. Photogramm Eng Remote Sensing 75(8):971-979

Wu F (1998) Polycentric urban development and land-use change in a transitional economy: the case of Guangzhou. Environ Plann A 30:1077-1100 
Wu F (2002) Calibration of stochastic cellular automata: the application to ruralurban land conversions. Int J Geogr Inf Sci 16(8):795-818

Wu H, Zhou L, Chi X, Li Y, Sun Y (2012) Quantifying and analyzing neighborhood configuration characteristics to cellular automata for land use simulation considering data source error. Earth Sci Inform 5(2):77-86

Yeh AG-O, Li X (2006) Errors and uncertainties in urban cellular automata. Comput Environ Urban 30(1):10-28

Zhang Q, He K, Huo H (2012) Cleaning China's air. Nature 484(7393):161-162

Zhou Y, Li X, Asrar GR, Smith SJ, Imhoff M (2018) A global record of annual urban dynamics (1992-2013) from nighttime lights. Remote Sens Environ 219:206220

Zhou Y, Smith SJ, Elvidge CD, Zhao K, Thomson A, Imhoff M (2014) A clusterbased method to map urban area from DMSP/OLS nightlights. Remote Sens Environ 147:173-185

Zhou Y, Smith SJ, Zhao K et al (2015) A global map of urban extent from nightlights. Environ Res Lett 10(5):1-11

\section{Publisher's Note}

Springer Nature remains neutral with regard to jurisdictional claims in published maps and institutional affiliations.

\section{Submit your manuscript to a SpringerOpen ${ }^{\mathcal{O}}$ journal and benefit from:}

- Convenient online submission

- Rigorous peer review

- Open access: articles freely available online

High visibility within the field

- Retaining the copyright to your article

Submit your next manuscript at $\boldsymbol{\nabla}$ springeropen.com 